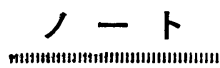

\title{
Determination of the Relative Content of U-235 in Enriched Uranium by Alpha-Gamma Coincidence Method Using a Gridded Ionization Chamber
}

\author{
Mitsuhiro Mryajima and Tadayoshi Doke* \\ Department of Physics, School of Science and Engineering, \\ Waseda University, Kikui-cho, Shinjuku-ku, Tokyo \\ *Institute for Nuclear Study, University of Tokyo Tanashi-shi, Tokyo
}

Received January 20, 1968

The alpha-gamma coincidence technique has been often used for the absolute measurement of alpha-activity and the identification of a particular nucleus in the presence of other alpha-active isotopes. For the measurement of weak alpha-source, an alpha-gamma coincidence system composed of a gridded ionization chamber and a $\mathrm{NaI}$ scintillation counter is superior to a conventional detector system of two scintillation counters, since the source area and the solid angle for alpha-particle detection in the gridded chamber are larger than those in the scintillation counter. In such a coincidence system, generally, the voltage pulse induced on the collector of the chamber is used for the alpha-gamma coincidence. The resolving time for the fast coincidence between the collector pulse and the gamma-pulse must be kept longer than one micro-second, because the average delay of the start time of the collector pulse relative to the alpha-decay is about one micro-second and furthermore the delay fluctuates over a few tenth of a micro-second depending on the emission angle of alpha-particle.

Bochagov, Vorob'ev and Komar'1),2) found that the height of the voltage pulse induced on the cathode in a gridded chamber depends on the emission angle of alpha-particle in the same formula as that of the collector pulse in a parallel plate chamber ${ }^{3}$ and suggested a possibility of the measurement of alpha-gamma angular correlation using the cathode pulse. Such a cathode pulse rises up at the same time as the emission of alpha-particle. Accordingly, if the cathode pulse is used in coincidence with the pulse due to gamma-rays, the resolving time for fast coincidence can be easily reduced to the order of 0.1 micro-sceond. From this view-point, we tried to determine the relative content of U-235 in a slightly enriched uranium using the cathode pulse for alphagamma coincidence in the detection system composed of a gridded chamber and a $\mathrm{NaI}$ scintillation counter.

Fig. 1 shows a block diagram of the detection

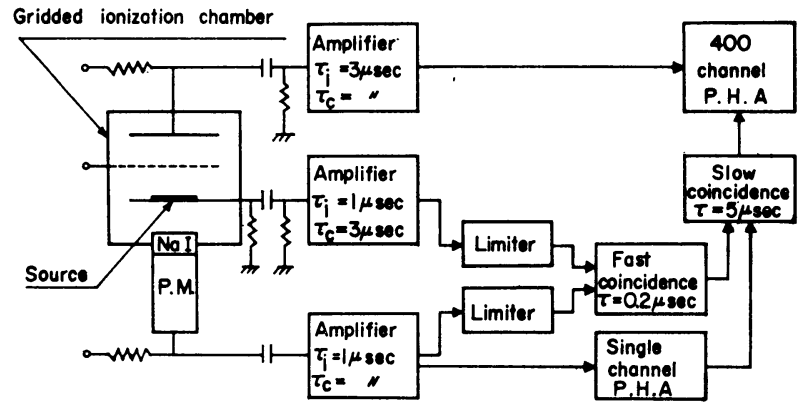

Fig. 1 Schematic diagram of detection system for $\alpha-\gamma$ coincidence measurement. 
system and its electronic circuit. The gridded chamber used in this system has a distance of $30 \mathrm{~mm}$ between the cathode and the grid, and $30 \mathrm{~mm}$ also between the grid and the collector. The grid is constructed with tungsten wires $0.1 \mathrm{~mm}$ in diameter and $1 \mathrm{~mm}$ apart, and the shielding inefficiency is estimated to be $0.62 \%$. The stainless source plate of $60 \mathrm{~mm}$ in diameter is placed at the center of the cathode. This chamber was filled with a gas mixture of $85 \%$ argon and $15 \%$ methane and operated at three atm. A NaI crystal of $1 \frac{1}{2}$ in. in diameter $\times 1 \mathrm{in}$. in height is placed at the distance of $70 \mathrm{~mm}$ below the alpha-source.

For the collector pulse the amplifier with $3 \mu \mathrm{sec}$ integrating time constant $\left(\tau_{\mathrm{i}}\right)$ and $3 \mu \mathrm{sec}$ clipping time constant $\left(\tau_{\mathrm{c}}\right)$, for the cathode pulse the amplifier with $\tau_{\mathrm{i}}=1 \mu \mathrm{sec}$ and $\tau_{\mathrm{c}}=3$ $\mu \mathrm{sec}$, and for the scintillation pulse the amplifier with $\tau_{\mathrm{i}}=1 \mu \mathrm{sec}$ and $\tau_{\mathrm{c}}=1 \mu \mathrm{sec}$ is used respectively. The resolving times of the fast

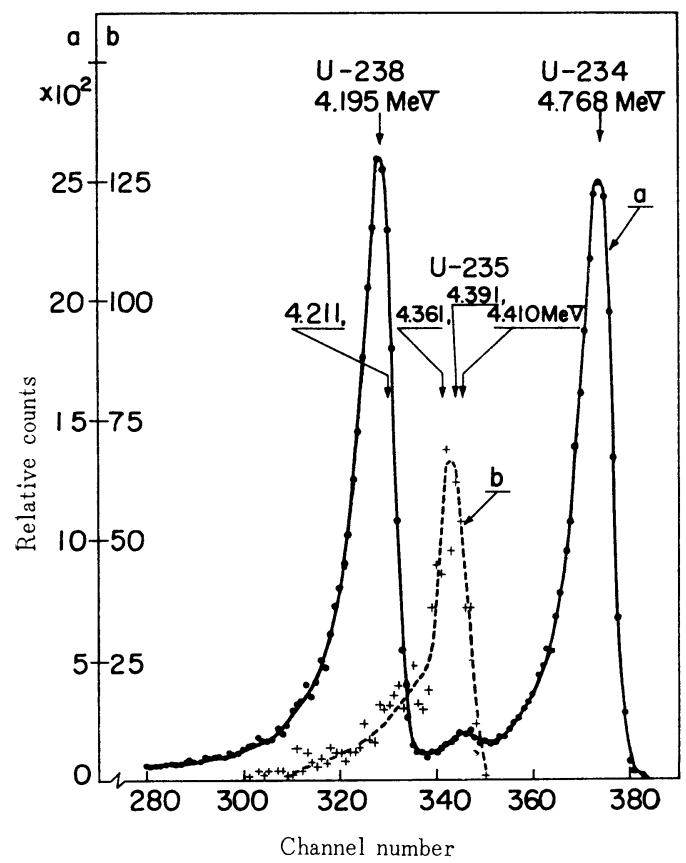

Fig. 2 a: Total energy spectrum of the alphaparticles emitted from natural uranium source.

b: Energy spectrum of the alpha-particles from the natural uranium source coincident with the gamma-rays in the energy region of $175 \mathrm{keV}$ to $225 \mathrm{keV}$.

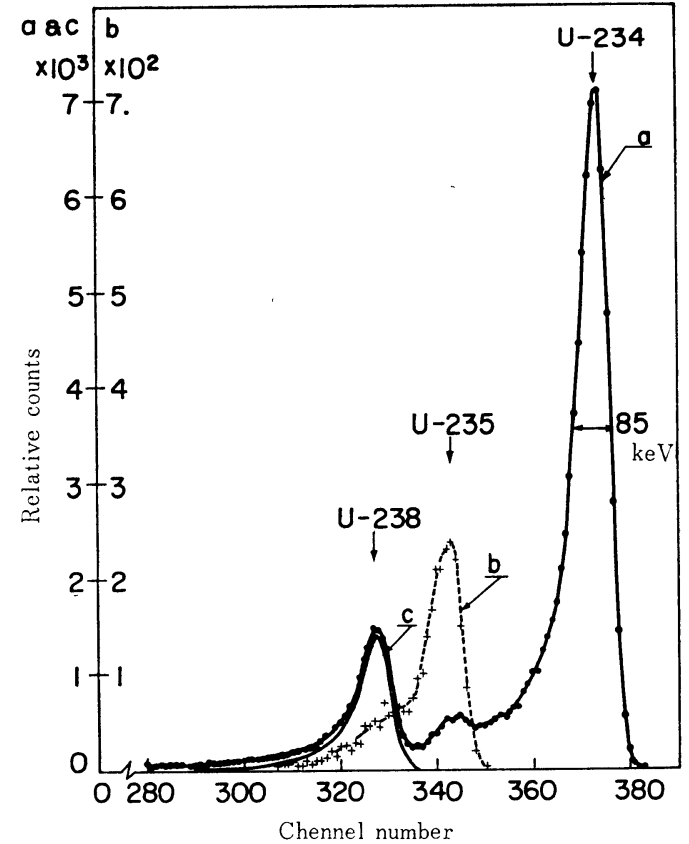

Fig. 3 a: Total energy spectrum of the alphaparticles emitted from enriched uranium source.

b: Energy spectrum of the alpha-particles from the enriched uranium source coincident with the gammarays in the energy region of 175 $\mathrm{keV}$ to $225 \mathrm{keV}$.

c: Estimated energy spectrum of U-238 for the enriched uranium source.

and slow coincidence circuit are designed to be $0.2 \mu \mathrm{sec}$ and $5 \mu \mathrm{sec}$, respectively.

The relative content of U-235 in a slightly enriched uranium was determined from the energy spectrum of the alpha-particles coincident with the gamma-rays following alphadecay of U-235. Two energy regions of the gamma-rays, (i) $125 \mathrm{keV}$ to $175 \mathrm{keV}$ and (ii) 175 $\mathrm{keV}$ to $225 \mathrm{keV}$, were selected for the alphagamma coincidence. In both cases, the random coincident counts were less than 2 per cent of the net coincident counts. Figs. 2 and 3 show the energy spectra of the alpha-particles in coincidence with the gamma-rays in the energy interval $175 \mathrm{keV}$ to $225 \mathrm{keV}$ and without coincidence for the natural, and enriched uranium sample, respectively. Assuming that the natural isotope abundance ratio of uranium U-238: U-235: U-234 is $0.9928: 0.00715: 0.000058^{4}$, the 
detection efficiencies for alpha-particles emitted from U-235 in the uranium source were estimated to be $2.79 \times 10^{-3}$ for the case (i) and $5.28 \times$ $10^{-3}$ for the case (ii). Using these detection efficiencies, the number of U-235 atoms included in the enriched uranium sample can be easily obtained. Also, the number of U-238 atoms can be estimated from the energy of spectrum of the enriched sample. From the results of these estimates, the ratio of U-235 to U-238 in the enriched uranium was determined to be $(4.42 \pm 0.18) \times 10^{-2}$ from the case (i) and $(4.35 \pm 0.12) \times 10^{-2}$ from the case(ii), respectively. These ratios are in good agreement with the value $(4.4614 \pm 0.0005) \times 10^{-2}$ determined by a mass-spectrometer.

\section{References}

1) B.A. Bochagov, A.A. Vorob'ev and A.P. Komar: Izv. Akad. Nauk SSSR, Ser. Fiz., 20, 1455 (1956)

2) B.A. Bochagov, A.A. Vorob'ev and A.P. Komar: Zh. Tekhn. Fiz., 27, 1575 (1957)

3) C. Valladas, J. Teillac, P. Falk-Vairant et P. Benoist: J. Phys. Radium, 16, 125(1955)

4) D. Strominger, J.M. Hollander and G.T Seaborg: Revs. Mod. Phys., 30, 585 (1958) 